\title{
Convergents and irrationality measures of logarithms
}

Tanguy Rivoal

\begin{abstract}
We prove new irrationality measures with restricted denominators of the form $\mathrm{d}_{\lfloor\nu m\rfloor}^{s} B^{m}$ (where $B, m \in \mathbb{N}, \nu>0, s \in\{0,1\}$ and $\left.\mathrm{d}_{m}=\operatorname{lcm}\{1,2, \ldots, m\}\right)$ for values of the logarithm at certain rational numbers $r>0$. In particular, we show that such an irrationality measure of $\log (r)$ is arbitrarily close to 1 provided $r$ is sufficiently close to 1 . This implies certain results on the number of non-zero digits in the $b$-ary expansion of $\log (r)$ and on the structure of the denominators of convergents of $\log (r)$. No simple method for calculating the latter is known. For example, we show that, given integers $a, c \geq 1$, for all large enough $b, n$, the denominator $q_{n}$ of the $n$-th convergent of $\log (1 \pm a / b)$ cannot be written under the form $\mathrm{d}_{\lfloor\nu m\rfloor}^{s}(b c)^{m}$ : this is true for $a=c=1, b \geq 12$ when $s=0$, resp. $b \geq 2$ when $s=1$ and $\nu=1$. Our method rests on a detailed diophantine analysis of the upper Padé table $([p / q])_{p \geq q \geq 0}$ of the function $\log (1-x)$. Finally, we remark that worse results (of this form) are currently provable for the exponential function, despite the fact that the complete Padé table $([p / q])_{p, q \geq 0}$ of $\exp (x)$ and the convergents of $\exp (1 / b)$, for $|b| \geq 1$, are well-known, for example.
\end{abstract}

\section{Introduction and statement of the results}

In this article, we address the following question, amongst others: given a rational $r>0, r \neq 1$ and an integer $b \geq 2$, what bound can be put on $\lambda(b, r)>0$, defined as the infimum of the positive $\lambda$ such that, for all integers $u$ and $m \gg_{b, r, \lambda} 1$, we have

$$
\left|\log (r)-\frac{u}{b^{m}}\right| \geq \frac{1}{b^{\lambda m}}
$$

2000 Mathematics Subject Classification: Primary 11J82 ; Secondary 11J60.

Keywords: Irrationality measures with restricted denominators, Convergents, Padé approximants of the logarithm. 
Such an irrationality measure with restricted denominators is not only interesting for itself but it is also useful for studying normality or, less ambitiously and more pragmatically, bounding the counting function of non-zero digits in the $b$-ary expansion $\sum_{j=-k}^{\infty} d_{j}(\alpha) / b^{j}\left(\right.$ with $\left.d_{j}(\alpha) \in\{0,1, \ldots, b-1\}\right)$ of an irrational number $\alpha$, such as $\log (r)$. If we denote by $\left(u_{n} / b^{n}\right)_{n}$ the sequence of rational approximations to $\alpha$ deduced from the $b$-ary expansion of $\alpha$, then we have $\left|b^{n} \alpha-u_{n}\right| \leq 1$, which implies that $\lambda(b, r) \geq 1$.

If $\alpha$ is absolutely normal, as $\log (r)$ is believed to be, then there even exists an unbounded function $\varphi_{\alpha}(n)$ such that $\left|b^{n} \alpha-u_{n}\right| \leq b^{-\varphi_{\alpha}(n)}$, because there are infinitely many arbitrarily long sequences of zeros in the $b$-ary expansion of $\alpha$.

In the opposite direction, if $\alpha$ is normal, there does not exist a $\delta(\alpha)>0$ such that, for all $u \in \mathbb{Z}$ and all $m \gg_{\alpha, b} 1$, we have $\left|b^{m} \alpha-u\right| \leq b^{-\delta(\alpha) m}$ and it is likely that $\lambda(b, r)=1$. Hence, if we find a value of $\lambda>1$ satisfying (1.1) close to 1 , this gives us evidence for the normality of $\log (r)$. Something like this is true but the result obtained is very weak. Indeed, if we set $\log (r)=$ $\sum_{j=1}^{\infty} \eta_{j} / b^{e_{j}}$ with $\eta_{j} \in\{1, \ldots, b-1\}$ and $\left(e_{j}\right)_{j}$ a strictly increasing sequence of integers, the bound (1.1) implies that $e_{j+1} \leq \lambda e_{j}+\mathcal{O}(1)$. Therefore knowing that $1<\lambda<+\infty$ only implies that $e_{j} \ll \lambda^{j}$ as $N \rightarrow+\infty$ or, in other words, that

$$
\#\left\{j \leq N: d_{j}(\log (r)) \neq 0\right\} \geq \log (N) / \log (\lambda)+\mathcal{O}(1)
$$

whereas one expects that the left hand side of (1.2) should be equivalent to $(b-1) N / b$. Thus, to obtain a better lower bound in (1.2), rather than $\lambda(b, r)$, one should look for an explicit function $\psi_{r}$ such that $\psi_{r}(m)=o(m)$ and for all $u \in \mathbb{Z}$ and all $m \gg_{b, r} 1,\left|\log (r)-u / b^{m}\right| \geq 1 / b^{m+\psi_{r}(m)}$.

A related result is the following (special case of a more general) theorem of Ridout [18]: for any real irrational algebraic numbers $\alpha$, all $\varepsilon>0$, all integers $b \geq 2$, all $u \in \mathbb{Z}$ and all $m \geq 1$, we have $\left|b^{m} \alpha-u\right| \gg_{\varepsilon, b} b^{-\varepsilon m}$. Unfortunately, it is ineffective -the dependence of the implicit constants on $\varepsilon$ is not known- and Ridout's theorem does not provide something asymptotically better than (1.2) for $\#\left\{j \leq N: d_{j}(\alpha) \neq 0\right\}\left(^{1}\right)$. In order to solve certain Diophantine equations, Beukers [6], and then Bauer and Bennett [5], proved weaker but completely effective bounds of Ridout's type for quadratic numbers: the present work is partly inspired by Beukers' original method but is more systematic.

\footnotetext{
${ }^{1}$ An important improvement was recently obtained by Bailey et al in [3]: in base $b=2$, we have $\#\left\{j \leq N: d_{j}(\alpha)=1\right\} \gg N^{1 / D}$, where $D$ is the degree of $\alpha$. It would be very interesting to adapt their method to other types of real numbers.
} 
Nevertheless, proving that $\lambda(b, r)<2$ has an interesting consequence. Let $\left(p_{n} / q_{n}\right)_{n}$ denote the sequence of convergents of $\log (r)$ : we have that $\left|q_{n} \log (r)-p_{n}\right|<q_{n}^{-1}$ for all $n$ and, if $\lambda(b, r)<2$, we can conclude that, for all $n \gg_{b, r} 1$, the denominator $q_{n}$ cannot be an integral power of $b$. Although this result does not say what the convergents of $\log (r)$ are, it does at least say something about what they aren't.

To state our results, we first need to introduce some notations. From now on, we suppose that $a, b \in \mathbb{Z}, \kappa, x \in \mathbb{R}$ satisfy $\kappa \geq 1,0<|x|<1, b \geq 2$ and $0<|a / b|<1$. Let

$$
r_{0}=r_{0}(\kappa, x)=\frac{1}{2 \kappa|x|}\left((\kappa-1)(x-1)+\sqrt{(\kappa-1)^{2}(1-x)^{2}+4 \kappa(1-x)}\right)
$$

and

$$
t_{0}=t_{0}(\kappa, x)=\frac{1}{2 \kappa x}\left(\kappa(1+x)+1-x-\sqrt{(\kappa(1+x)+1-x)^{2}-4 \kappa^{2} x}\right)
$$

Let also

$$
\begin{aligned}
& \beta=\beta(\kappa, x)=\frac{\left(1+|x| r_{0}\right)^{\kappa}\left(1-x+|x| r_{0}\right)}{|x| r_{0}} \\
& \text { and } \rho=\rho(\kappa, x)=|x|^{\kappa+1} \frac{t_{0}^{\kappa}\left(1-t_{0}\right)}{1-x t_{0}},
\end{aligned}
$$

which satisfy $b \beta(\kappa, a / b) \geq 1\left({ }^{2}\right)$ and $\rho>0$.

Let $\mathscr{L}(a, b)=\left\{\kappa \geq 1: b^{\kappa} e^{\kappa} \rho(\kappa, a / b)<1\right\}$; we do not exclude the possibility that this set might be empty. For any $\kappa \in \mathscr{L}(a, b)$, we necessarily have $b \rho<1$ and $b e^{\kappa} \rho<1$, hence $\log (\beta / \rho)(=\log (b \beta / b \rho))$ and $-\log \left(b e^{\kappa} \rho\right)$ are positive and the quantity

$$
L(\kappa, a, b)=\frac{\log (\beta(\kappa, a / b) / \rho(\kappa, a / b))}{-\log \left(b e^{\kappa} \rho(\kappa, a / b)\right)}
$$

is well-defined and positive.

The best possible upper bound $\Lambda(a, b, c)$ of $\lambda(b c, 1-a / b)$ that we prove in this article is given in Theorem 1. A more explicit version is produced in Theorem 2. All the constants involved in the various symbols "》" spread in the text, in principle, can be given explicitly if really necessary; we use the notation $\gg_{e_{1}, e_{2}, \ldots}$ to indicate that these constants depend at most on $e_{1}, e_{2}$, etc.

\footnotetext{
${ }^{2}$ The fact that $b \beta(\kappa, a / b) \geq 1$ is not obvious from this definition and is proved in Lemma 2 in Section 2.
} 
Theorem 1. Let $a, b, c$ be integers such that $0<|a / b|<1, b \geq 2, c \geq 1$ and $\mathscr{L}(a c, b c) \neq \emptyset$. Let $\varepsilon$ be any positive real number. Then, for any $u \in \mathbb{Z}$ and any $m \gg_{\varepsilon, a, b, c} 1$, we have

$$
\left|\log \left(1-\frac{a}{b}\right)-\frac{u}{(b c)^{m}}\right| \geq \frac{1}{(b c)^{(\Lambda(a, b, c)+\varepsilon) m}} .
$$

with

$$
\Lambda(a, b, c)=\inf _{\kappa \in \mathscr{L}(a c, b c)} L(\kappa, a c, b c) .
$$

Remarks. 1) Since $\Lambda(a, b, c)=\Lambda(a c, b c, 1)$, it is enough to prove the theorem for $c=1$.

2 ) It is not obvious that $\Lambda(a, b, c)$ can ever be strictly less than 2 . However, Theorem 2 shows that $L(\kappa, a c, b c)$ takes values arbitrarily close to 1 when $b \gg_{a, c} 1$.

3) Note that $L(1, a, b)$ is the irrationality measure for $\log (1-a / b)$ obtained by Alladi and Robinson [2]: thus $\Lambda(a, b, 1)$ is smaller than this classical bound. Furthermore, for a given $\kappa \in \mathscr{L}(a, b), \kappa>1, L(\kappa, a, b)$ is strictly smaller than the general irrationality measure

$$
\frac{\log (\beta(\kappa, a / b) / \rho(\kappa, a / b))}{-\log \left(b^{\kappa} e^{\kappa} \rho(\kappa, a / b)\right)}
$$

which can be deduced by the methods of this paper: see the discussion in Section 4.

We now state a more explicit (but less precise) form of Theorem 1.

Theorem 2. Let $a, b, c$ be integers such that $0<|a / b|<1, b \geq 2, c \geq 1$.

(i) If $a<0$ and $1+2 \log |a|+\log (c) \leq \log (b)$, then

$$
K=\frac{\log (b /|a|)}{1+\log |a c|} \in \mathscr{L}(a c, b c)
$$

and

$$
1 \leq L(K, a c, b c) \leq 1+\frac{K \log (2)+\log (2-a / b)+K+\log (b c)}{\log \left(K^{-K}(K+1)^{K+1}|b / a|^{K+1}\right)-K-\log (b c)} .
$$

(ii) If $a>0$ and $1+2 \log (a)+\log (c) \leq \log (b)+\log \sqrt{1-a / b}$, then

$$
k=\frac{\log (b / a)+\log \sqrt{1-a / b}}{1+\log (a c)} \in \mathscr{L}(a c, b c)
$$


and

$$
\begin{aligned}
1 & \leq L(k, a c, b c) \\
& \leq 1+\frac{k \log (2)+\log (2-a / b)+k+\log (b c)}{\log \left((2 k)^{-k}(2 k+1)^{k+1 / 2}(b / a)^{k+1}(1-a / b)^{1 / 2}\right)-k-\log (b c)} .
\end{aligned}
$$

(iii) As $b \rightarrow+\infty$, we have

$$
1 \leq \Lambda(a, b, c) \leq 1+\frac{2+\log (2|a c|)}{\log (b c)}+\mathcal{O}\left(\frac{1}{\log ^{2}(b)}\right)
$$

Remark. As it will be clear from the proof of Theorem 2, better bounds could be obtained at the cost of more complicated formulas.

As anticipated, an interesting consequence of point ( $\mathrm{iii}$ ) of Theorem 2 is the following result.

Theorem 3. Let $a, b, c$ be integers such that $0<|a / b|<1, b \geq 2, c \geq 1$. Let $\left(p_{n} / q_{n}\right)_{n \geq 0}$ denotes the infinite sequence of convergents of $\log (1-a / b)$. Then, for all $b \gg_{a, c} 1$ and all $n \gg_{a, b, c} 1$, the denominator $q_{n}$ is not an integral power of bc.

Remarks. 1) For similar results, see (iii) in Theorem 4 and the comments around Equation (7.1) in Section 7.

2) It is notoriously difficult to compute the convergents of numbers like $\log (2)$ efficiently, i.e, without going back to the definition of a continued fraction and starting with more and more accurate approximations of $\log (2)$ (which is cheating).

3) There exists a simple algorithm, due to Shanks, which enables us to compute the continued fractions of $\ell=\log (a) / \log (b)$ for any integers $a>b>1$, in which only rational numbers intervene and no approximation to $\ell$ is ever calculated: see [14] for details. However, the involved integers become quickly very large and this algorithm seems to be interesting more from a theoretical than a practical point of view. Furthermore, it does not give much information about what are or are not the convergents of $\ell$. This is also the case of certain algorithms deviced to compute efficiently the continued fractions of real algebraic numbers: see [8] for a survey.

In the particular case $a=c=1$, we have $\Lambda(1, b, 1)<2$ for $|b| \geq 12$ : the formulas in Theorem 2 proves this for $b \leq-27$ and $b \geq 37$, the remaining cases being proved using Theorem 1 . Hence, in Theorem 3, the condition $b \gg_{a, c} 1$ can be replaced by $|b| \geq 12$ when $a=c=1$. 
The following table presents approximations for values of $\Lambda(a, b, c)$. In the third column, when $2 \leq|a| \leq 5$, the value of $b$ is the smallest such that $\Lambda(a, b, 1)<2$. The infimum $\Lambda(a, b, c)$ is generally obtained at a $\kappa>1$, which justifies a posteriori our general construction. For example, for $a=$ $c=1, b=4$, we have $\kappa \approx 3.56$.

\begin{tabular}{|cc|cc|cc|cc|}
\hline$b$ & $\Lambda(1, b, 1)$ & $b$ & $\Lambda(1, b, 1)$ & $(a, b)$ & $\Lambda(a, b, 1)$ & $(a, b, c)$ & $\Lambda(a, b, c)$ \\
\hline 2 & 4.6221 & -2 & 3.5474 & $(2,61)$ & 1.9989 & $(1,31,1)$ & 1.6917 \\
\hline 3 & 3.5287 & -3 & 3.2131 & $(-2,60)$ & 1.9995 & $(1,31,2)$ & 1.9934 \\
\hline 4 & 3.0045 & -4 & 2.9016 & $(3,179)$ & 1.9986 & $(-1,30,1)$ & 1.6978 \\
\hline 5 & 2.6684 & -5 & 2.6154 & $(-3,177)$ & 1.9992 & $(-1,30,2)$ & 1.9995 \\
\hline 6 & 2.4661 & -6 & 2.4320 & $(4,394)$ & 1.9997 & $(1,60,1)$ & 1.5684 \\
\hline 7 & 2.3290 & -7 & 2.3051 & $(-4,392)$ & 1.9994 & $(1,60,2)$ & 1.8089 \\
\hline 8 & 2.2291 & -8 & 2.2113 & $(5,737)$ & 1.9998 & $(1,60,3)$ & 1.9969 \\
\hline 9 & 2.1523 & -9 & 2.1385 & $(-5,734)$ & 1.9997 & $(-1,59,1)$ & 1.5706 \\
\hline 10 & 2.0912 & -10 & 2.0801 & $\left(1,2^{8}\right)$ & 1.4071 & $(-1,59,2)$ & 1.8111 \\
\hline 11 & 2.0411 & -11 & 2.0320 & $\left(1,3^{9}\right)$ & 1.2184 & $(-1,59,3)$ & 1.9992 \\
\hline 12 & 1.9992 & -12 & 1.9915 & $\left(-1,5^{7}\right)$ & 1.1901 & $(2,197,2)$ & 1.9997 \\
\hline 13 & 1.9634 & -13 & 1.9568 & $\left(1,10^{7}\right)$ & 1.1305 & $(-3,3493,5)$ & 1.9999 \\
\hline
\end{tabular}

The proof of Theorems 1 and 2 will be given in Sections 3 and 5, respectively. Baker's theory of linear form of logarithms is not sharp enough to prove any of these results, even assuming such general conjectures as LangWaldschmidt [20, p. 11]. Instead, we resort to the less general but much sharper theory of Padé approximants of $\log (1-x)$, which we recall in details in the Lemmas 1 and 2 in Section 2.

The irrationality measures of logarithm found in the litterature are proved using diagonal Padé approximants $[n / n]$ (Baker, Alladi and Robinson [2]) or slightly modified versions of the same (Rukhadze [19], Hata [12], Heimonen et al [13]); none deals with irrationality measures with restricted denominators. To treat this case, we will use Padé approximants $[p n / q n]$ with $p=\kappa q$ substantially larger than $q$ in order to get a crucial asymmetric term $b^{(p-q) n}$ in the estimates for $\log (1-a / b)$. This trick was apparently first used by Beukers [6].

Another noteworthy feature of the Padé approximants $[p n / q n]$ of $\log (1-x)$ is the presence of a factor $\mathrm{d}_{p n}$, where $\mathrm{d}_{m}=\operatorname{lcm}(1,2, \ldots, m)$. It is fundamentally different from $b^{(p-q) n}$ but it also intervenes in an asymmetric way (see eq. (2.1) of Lemma 1 in Section 2) and provides some non-trivial diophantine information which we summarise in the following result, whose proof in Section 6 will only be sketched since it is very similar to those of Theorems 1, 2 and 3. 
Theorem 4. Let $a, b, c$ be integers such that $b, c \geq 1$ and $0<|a / b|<1$.

(i) If eb $\rho(1, a / b)<1$, then for all $u \in \mathbb{Z}$ and $m \in \mathbb{N}$, we have

$$
\left|\log \left(1-\frac{a}{b}\right)-\frac{u}{\mathrm{~d}_{m}}\right| \gg_{a, b} \frac{1}{\mathrm{~d}_{m}^{2-\frac{2 \log |a|}{\log (b \rho(1, a / b))}}} .
$$

(ii) Let $\nu \in \mathbb{R}, \nu>0$, and suppose that

$\mathscr{D}(a, b, \nu)=\left\{\kappa \geq 1: e^{\kappa} b^{\kappa / \nu+1} \rho(\kappa, a / b)<1\right.$ and $\left.e^{(\kappa-1) \nu} b^{\kappa} \rho(\kappa, a / b)<1\right\} \neq \emptyset$.

Then, for all $\varepsilon>0, u \in \mathbb{Z}$ and $m \gg_{\varepsilon, \nu, a, b, c} 1$, we have

$$
\left|\log \left(1-\frac{a}{b}\right)-\frac{u}{\mathrm{~d}_{\lfloor\nu m\rfloor}(b c)^{m}}\right| \geq \frac{1}{\left(\mathrm{~d}_{\lfloor\nu m\rfloor}(b c)^{m}\right)^{D(a c, b c, \nu)+\varepsilon}} .
$$

where

$$
D(a, b, \nu)=\inf _{\kappa \in \mathscr{D}(a, b, \nu)} \frac{\log (\beta(\kappa, a / b) / \rho(\kappa, a / b))}{-\log (b \rho(\kappa, a / b))} \rightarrow 1 \quad \text { as } b \rightarrow+\infty .
$$

(iii) For all $b \gg_{a, c, \nu} 1, n \gg_{a, b, c, \nu} 1$ and $m \in \mathbb{N}$, we have that $q_{n} \neq \mathrm{d}_{\lfloor\nu m\rfloor}(b c)^{m}$, where $q_{n}$ is the denominator of the $n$-th convergent of $\log (1-a / b)$.

Remarks. 1) In (i), we could have stated a result for $\kappa \geq 1$ but numerically it seems that the best value is always attained at $\kappa=1$. The particular case when $a= \pm 1$ of $(1.8)$ reads $\left|\log (1-1 / b)-u / \mathrm{d}_{m}\right| \gg_{b} 1 / \mathrm{d}_{m}^{2}$ for all $|b| \geq 2$. It is difficult to compute the implicit constant accurately and a rough estimate gives a value smaller than 1 ; for this reason, we cannot deduce from (1.8) a result similar to $(\mathrm{iii})$ in this case. However, as indicated by a referee, such a result is true when $b=-1$ by a theorem of Dubitskas [10] which implies that $\left|\log (2)-u / \mathrm{d}_{m}\right| \gg \log \left(\mathrm{d}_{m}\right) / \mathrm{d}_{m}^{2}$, a stronger inequality than (1.8) in this case.

2) With $a=c=\nu=1$ in (1.9) for instance, we obtain that, for all $u \in \mathbb{Z}$ and $m \gg 1$,

$$
\left|\log (2)-\frac{u}{\mathrm{~d}_{m} 2^{m}}\right| \geq \frac{1}{\left(\mathrm{~d}_{m} 2^{m}\right)^{1.948967}} .
$$

Finally, it is easy to see why it is possible to improve irrationality measures when considering special denominators rather than arbitrary ones: $\left(b^{m}\right)_{m \geq 0}$ and $\left(\mathrm{d}_{m}\right)_{m \geq 1}$ are examples of sequences $\left(u_{m}\right)_{m}$ satisfying $u_{m} \mid u_{n}$ for $m \leq n$. More precisely, to estimate the difference $\log (1-a / b)-u / v$ using the methods of this article, we need to find a lower bound of a certain difference $D=\left|u / v-U /\left(\mathrm{d}_{m} b^{k} V\right)\right|$ between two distinct rationals $(m, k \in \mathbb{N}$, $u, v, U, V \in \mathbb{Z})$. When $v$ could be anything, the best we can say is that, trivially, $D \geq\left(\mathrm{d}_{m} b^{k} V v\right)^{-1}$; however, if we know in advance that $v=\mathrm{d}_{M} b^{K}$ then we can improve our trivial bound to $D \geq\left(\mathrm{d}_{\max (M, m)} b^{\max (K, k)} V\right)^{-1}$ and thus save a crucial factor of $\mathrm{d}_{\min (M, m)} b^{\min (K, k)}$ in the process. 


\section{The upper Padé table of the logarithm}

Let $p, q$ be integers such that $p \geq q \geq 0$. There exist non-zero polynomials $A_{p, q}(X)$ and $B_{p, q}(X)$ in $\mathbb{Q}[X]$ of degree at most $p$ and $q$ respectively such that the order at $x=0$ of the (entire or formal) series

$$
R_{p, q}(X)=B_{p, q}(X) \log (1-X)-A_{p, q}(X)
$$

is at least $p+q+1$. These polynomials, which are unique $\left({ }^{3}\right)$ up to a multiplicative constant, define the Padé approximant $A_{p, q} / B_{p, q}=[p / q]$ of $\log (1-X)$ and we summarize their properties in the following lemma (which belongs to folklore).

Lemma 1. ( $i)$ In the above conditions, when $p \geq q$, we can choose the multiplicative constant such that the following conditions are satisfied:

$$
\begin{gathered}
\mathrm{d}_{p} A_{p, q}(x) \in \mathbb{Z}[x], \quad B_{p, q}(x) \in \mathbb{Z}[x], \\
x^{q} B_{p, q}(1 / x)=x^{q-p}\left(x^{p}(1-x)^{q}\right)^{(q)} / q !
\end{gathered}
$$

and

$$
R_{p, q}(x)=x^{p+q+1} \int_{0}^{1} \frac{t^{p}(1-t)^{q}}{(x t-1)^{q+1}} \mathrm{~d} t .
$$

(ii) There exists a non-zero constant $c_{p, q}$ such that

$$
A_{p+1, q+1}(x) B_{p, q}(x)-A_{p, q}(x) B_{p+1, q+1}(x)=c_{p, q} x^{p+q+1} .
$$

(iii) For all $x \in(-1,1), B_{p, q}(x) \neq 0$.

Remark. Unfortunately, no such formulas are known when $p<q$. This rules out the possibility of proving results like Theorems 1, 2, 3 and 4 for the function $1 / \log (1-x)$ by similar methods.

Proof: $(i)$ We define the Pocchammer symbol by $(u)_{n}=u(u+1) \cdots(u+$ $n-1)$ and start with the hypergeometric series

$$
\begin{aligned}
\widehat{R}_{p, q}(x)=(-1)^{q+1} \sum_{k=1}^{\infty} \frac{(k-q)_{q}}{(k+p-q)_{q+1}} x^{p+k} & \\
& =\frac{(-1)^{q+1} x^{p+q+1}}{\left(\begin{array}{c}
p+q \\
p
\end{array}\right)(p+q+1)}{ }_{2} F_{1}\left[\begin{array}{c}
p+1, q+1 \\
p+q+2
\end{array} ; x\right],
\end{aligned}
$$

which converges for $|x| \leq 1, x \neq 1$ and has a zero of order $p+q+1$ at $x=0$.

\footnotetext{
${ }^{3}$ This is not always true for other power series $F$. However the fraction $[p / q]_{F}$ is unique: see [4] for the theory of Padé approximants.
} 
From the partial fractions expansion

$$
\frac{(k-q)_{q}}{(k+p-q)_{q+1}}=\sum_{j=0}^{q}(-1)^{j+q}\left(\begin{array}{c}
q \\
j
\end{array}\right)\left(\begin{array}{c}
p+j \\
q
\end{array}\right) \frac{1}{k+j+p-q},
$$

we obtain that

$$
\begin{aligned}
\widehat{R}_{p, q}(x) & =\sum_{j=0}^{q}(-1)^{j+1}\left(\begin{array}{c}
q \\
j
\end{array}\right)\left(\begin{array}{c}
p+j \\
q
\end{array}\right) \sum_{k=1}^{\infty} \frac{x^{p+k}}{k+p-q+j} \\
& =\sum_{j=0}^{q}(-1)^{j+1}\left(\begin{array}{c}
q \\
j
\end{array}\right)\left(\begin{array}{c}
p+j \\
q
\end{array}\right)\left(x^{q-j} \sum_{k=1}^{\infty} \frac{x^{k}}{k}-\sum_{k=1}^{j+p-q} \frac{x^{k+q-j}}{k}\right) \\
& =\widehat{B}_{p, q}(x) \log (1-x)-\widehat{A}_{p, q}(x),
\end{aligned}
$$

where

$$
\widehat{B}_{p, q}(x)=\sum_{j=0}^{q}(-1)^{j}\left(\begin{array}{c}
q \\
j
\end{array}\right)\left(\begin{array}{c}
p+j \\
q
\end{array}\right) x^{q-j} \in \mathbb{Z}[x]
$$

is of degree $q$ and

$$
\widehat{A}_{p, q}(x)=\sum_{j=0}^{q} \sum_{k=1}^{j+p-q}(-1)^{j+1}\left(\begin{array}{c}
q \\
j
\end{array}\right)\left(\begin{array}{c}
p+j \\
q
\end{array}\right) \frac{1}{k} x^{k+q-j} \in \mathrm{d}_{p}^{-1} \mathbb{Z}[x]
$$

is of degree $p$. Thus, we can define $A_{p, q}(x), B_{p, q}(x)$ and $R_{p, q}(x)$ as $\widehat{A}_{p, q}(x)$, $\widehat{B}_{p, q}(x)$ and $\widehat{R}_{p, q}(x)$ respectively. Classical computations can be used to prove the expected formulas for $B_{p, q}(x)$ and $R_{p, q}(x)$ : see [12, p. 100] and [13, pp. 186-187] for similar transformations.

(ii) We now turn our attention to the polynomial $C_{p, q}=A_{p+1, q+1} B_{p, q}-$ $A_{p, q} B_{p+1, q+1}$, whose degree is obviously at most $p+q+1$. Since $A_{p, q}=$ $B_{p, q} \log (1-x)-R_{p, q}$, we also have the identity $C_{p, q}=B_{p+1, q+1} R_{p, q}-$ $B_{p, q} R_{p+1, q+1}$, which implies that the order at zero of $C_{p, q}$ is at least $p+q+1$. Hence there exists a constant $c_{p, q}$ such that $C_{p, q}(x)=c_{p, q} x^{p+q+1}$. Explicit formulas for $B_{p, q}$ and $R_{p, q}$ yield the coefficients needed to compute $c_{p, q}$ : we have that

$$
(-1)^{q+1} c_{p, q}=\frac{\left(\begin{array}{l}
p+1 \\
q+1
\end{array}\right)}{\left(\begin{array}{c}
p+q \\
p
\end{array}\right)(p+q+1)}+\frac{\left(\begin{array}{l}
p \\
q
\end{array}\right)}{\left(\begin{array}{c}
p+q+2 \\
p+1
\end{array}\right)(p+q+3)} \neq 0 .
$$

(iii) We will prove something more, i.e. that the zeros of $B_{p, q}$ are all in $(1,+\infty)$. It follows from the expression $(2.2)$ for $B_{p, q}(x)$ that this is equivalent to proving that the zeros of the polynomial $x^{q-p}\left(x^{p}(1-x)^{q}\right)^{(q)} / q$ ! are 
all in $(0,1)$. There exists a classical proof based on Rolle's theorem. Instead, we propose the argument indicated by a referee. It is based on GaußLucas' theorem claiming that all roots of the derivative $P^{\prime}$ of a polynomial $P$ lie in the convex hull of the roots of $P$. Starting from the polynomial $P(x)=x^{p}(1-x)^{q}$, the roots of $P^{\prime}$ are thus all in $[0,1]$, hence this is also true for the roots of $P^{\prime \prime}$ and so on.

The proofs of our theorems are based on the asymptotic behaviour of the approximants $[p n+\eta / q n+\eta], \eta \in\{0,1\}$, when the integer $n$ tends to infinity. We use the functions $\beta$ and $\rho$ defined by (1.5) in the introduction.

Lemma 2. (i) Let $x$ such that $|x| \leq 1, x \neq 1$. Let $\eta$ denotes either 0 or 1 . We have

$$
\lim _{n \rightarrow+\infty}\left|R_{p n+\eta, q n+\eta}(x)\right|^{1 / q n}=\rho(p / q, x)
$$

and

$$
\limsup _{n \rightarrow+\infty}\left|B_{p n+\eta, q n+\eta}(x)\right|^{1 / q n} \leq \beta(p / q, x) .
$$

(ii) If $a, b \in \mathbb{Z}$ such that $|a / b|<1$ and $b \geq 1$, we have $b \beta(\kappa, a / b) \geq 1$ for all $\kappa \geq 1$.

Proof: $(i)$ We will give a detailed proof of the case $\eta=0$ and explain what must be changed when $\eta=1$. To simplify, we temporarily define $\kappa$ to be $p / q$.

From (2.3) in Lemma 1, we have

$$
R_{p n, q n}(x)=x^{(p+q) n+1} \int_{0}^{1} \frac{t^{p n}(1-t)^{q n}}{(x t-1)^{q n+1}} \mathrm{~d} t
$$

so that

$$
\lim _{n \rightarrow+\infty}\left|R_{p n, q n}(x)\right|^{1 / n}=|x|^{p+q} \max _{t \in[0,1]} \frac{t^{p}(1-t)^{q}}{(1-x t)^{q}} .
$$

This maximum is attained at $\widehat{t}_{0}$ (which depends on $p, q, x$ ), defined to be the unique root in $(0,1)$ of the equation

$$
\frac{\mathrm{d}}{\mathrm{d} t}\left(\frac{t^{p}(1-t)^{q}}{(1-x t)^{q}}\right)=0
$$

It turns out that $\widehat{t}_{0}$ is a solution of the quadratic equation

$$
\kappa x t^{2}-(\kappa(1+x)+1-x) t+\kappa=0,
$$

which means that $\widehat{t}_{0}$ depends only on the quotient $\kappa=p / q$ : this is exactly the number $t_{0}$ defined in (1.4).

Finally, we have

$$
\lim _{n \rightarrow+\infty}\left|R_{p n, q n}(x)\right|^{1 / q n}=|x|^{\kappa+1} \frac{t_{0}^{\kappa}\left(1-t_{0}\right)}{1-x t_{0}}=\rho(\kappa, x)>0 .
$$


Using Cauchy's integral formula and some straightforward simplifications, we transform (2.2) in Lemma 1 for $B_{p n, q n}(x)$ in the following way:

$$
\begin{aligned}
B_{p n, q n}(x)=\frac{x^{(p+q) n+1}}{2 i \pi} \int_{\mathscr{C}_{1 / x, r}} & \frac{z^{p n}(1-z)^{q n}}{(x z-1)^{q n+1}} \mathrm{~d} z \\
= & \frac{(-x)^{-q n}}{2 i \pi} \int_{\mathscr{C}_{0, r}} \frac{(1+x z)^{p n}(1-x+x z)^{q n}}{z^{q n+1}} \mathrm{~d} z,
\end{aligned}
$$

where $\mathscr{C}_{M, r}$ denotes the circle of center $M$ and radius $r$. Thus, we have

$$
\limsup _{n \rightarrow+\infty}\left|B_{p n, q n}(x)\right|^{1 / n} \leq \frac{1}{|x|^{q}} \min _{r>0} \frac{(1+|x| r)^{p}(1-x+|x| r)^{q}}{r^{q}} .
$$

This minimum is obtained for $\widehat{r}_{0}$ (depending a priori on $p, q, x$ ) defined as the positive solution of the quadratic equation

$$
\kappa|x|^{2} r^{2}+(\kappa-1)(1-x)|x| r-(1-x)=0 .
$$

It follows that $\widehat{r}_{0}$ depends only on the quotient $\kappa=p / q$ and is exactly the number $r_{0}$ defined in (1.3). Finally, we obtain that

$$
\limsup _{n \rightarrow+\infty}\left|B_{p n, q n}(x)\right|^{1 / q n} \leq \frac{\left(1+|x| r_{0}\right)^{\kappa}\left(1-x+|x| r_{0}\right)}{|x| r_{0}}=\beta(\kappa, x) .
$$

When $\eta=1$, we use the formulas

$$
\begin{aligned}
& R_{p n+1, q n+1}(x)=x^{(p+q) n+3} \int_{0}^{1} \frac{t^{p n+1}(1-t)^{q n+1}}{(x t-1)^{q n+2}} \mathrm{~d} t \\
& B_{p n+1, q n+1}(x)=\frac{x^{(p+q) n+3}}{2 i \pi} \int_{\mathscr{C}_{1 / x, r}} \frac{z^{p n+1}(1-z)^{q n+1}}{(x z-1)^{q n+2}} \mathrm{~d} z
\end{aligned}
$$

and the proof follows in a similar way.

(ii) We know from (2.1) and (iii) in Lemma 1 that $\left|b^{q n} B_{p n, q n}(a / b)\right|$ is a non-zero integer, hence that $\left|b^{q n} B_{p n, q n}(a / b)\right|^{1 / q n} \geq 1$ : letting $n \rightarrow+\infty$ proves that $b \beta(p / q, a / b) \geq 1$ for all rational $p / q \geq 1$. Since the function $\kappa \mapsto \beta(\kappa, a / b)$ is continuous on $[1,+\infty)$, we have $b \beta(\kappa, a / b) \geq 1$ for all real $\kappa \geq 1$.

\section{Proof of Theorem 1}

Since the set $\mathscr{L}(a, b)$ is non-empty, it contains a rational $\kappa=p / q$; we may suppose that $p \geq 1$ and $q \geq 1$ are coprime, so we can use parameters depending on $H=H(\kappa)=\max (p, q)$. Throughout this section, we write $\beta(\kappa, a / b)$ and $\rho(\kappa, a / b)$ as $\beta$ and $\rho$, respectively. 
Since $a / b \in(-1,1)$, we have $B_{p n+\eta, q n+\eta}(a / b) \neq 0$ by the last statement of Lemma 1 and we can consider the fraction $\left(A_{p n+\eta, q n+\eta} / B_{p n+\eta, q n+\eta}\right)(a / b)$. Now, let $u \in \mathbb{Z}$ and $m \in \mathbb{N}$. We will have to compare the fractions $u / b^{m}$ and $\left(A_{p n, q n} / B_{p n, q n}\right)(a / b)$ : they might be equal, in which case Equation $(2.4)$ in Lemma 1 ensures that $u / b^{m} \neq\left(A_{p n+1, q n+1} / B_{p n+1, q n+1}\right)(a / b)$ (because $\left.a / b \neq 0\right)$. Thus, for any given $a, b, m, n, p, q, u$, there exists $\eta \in\{0,1\}$ (depending on these seven parameters) such that

$$
\frac{u}{b^{m}} \neq \frac{A_{p n+\eta, q n+\eta}(a / b)}{B_{p n+\eta, q n+\eta}(a / b)} \text {. }
$$

According to Lemma 2, the value of $\eta$ has no asymptotic influence: since our results are proved for large enough $n$, there is no loss of generality in supposing that we always have $\eta=0$ in (3.1) in order to simplify the notations.

From Lemma 1, we deduce that

$$
A_{p n, q n}(a / b)=\frac{U_{n}}{\mathrm{~d}_{p n} b^{p n}} \quad \text { and } \quad B_{p n, q n}(a / b)=\frac{V_{n}}{\mathrm{~d}_{p n} b^{q n}},
$$

with $U_{n}, V_{n} \in \mathbb{Z}$, and

$$
\left|\log \left(1-\frac{a}{b}\right)-\frac{U_{n}}{b^{(p-q) n} V_{n}}\right|=\left|\frac{R_{p n, q n}(a / b)}{B_{p n, q n}(a / b)}\right| .
$$

The definition of $V_{n}$ and (2.6) imply that

$$
\limsup _{n \rightarrow+\infty}\left|V_{n}\right|^{1 / q n}=b^{\delta}, \quad \text { with } \quad \delta=\frac{\kappa+\log (b)+\log (\beta)}{\log (b)} .
$$

We now distinguish two cases: $(p-q) n \geq m$ and $(p-q) n<m$.

First case. Suppose that $(p-q) n \geq m$; in particular, we necessarily have $\kappa>1$.

Since

$$
\frac{u}{b^{m}} \neq \frac{U_{n}}{b^{(p-q) n} V_{n}}
$$

we have

$$
\begin{aligned}
\left|\log \left(1-\frac{a}{b}\right)-\frac{u}{b^{m}}\right| & \geq\left|\frac{u}{b^{m}}-\frac{U_{n}}{b^{(p-q) n} V_{n}}\right|-\left|\log \left(1-\frac{a}{b}\right)-\frac{U_{n}}{b^{(p-q) n} V_{n}}\right| \\
& \geq \frac{1}{b^{(p-q) n}\left|V_{n}\right|}-\left|\frac{R_{p n, q n}(a / b)}{B_{p n, q n}(a / b)}\right| .
\end{aligned}
$$

Hence, provided that

$$
\left|2 b^{(p-q) n} V_{n} R_{p n, q n}(a / b)\right| \leq\left|B_{p n, q n}(a / b)\right|,
$$


we have

$$
\left|\log \left(1-\frac{a}{b}\right)-\frac{u}{b^{m}}\right| \geq \frac{1}{2 b^{(p-q) n}\left|V_{n}\right|} .
$$

Since $b^{(p-q) n} V_{n}$ is an (essentially) increasing function of $n$, it is natural to choose $n$ to be as small as possible subject to the constraint that $(p-q) n \geq m$, i.e. we set

$$
n=\left\lfloor\frac{m}{p-q}\right\rfloor+1=\left\lfloor\frac{m}{q(\kappa-1)}\right\rfloor+1 .
$$

We now verify that with this definition of $n$, the condition (3.3) is also satisfied for large $m$. Indeed, we can rewrite (3.3) as

$$
2 b^{\kappa n} \mathrm{~d}_{p n}^{1 / q n}\left|R_{p n, q n}(a / b)\right|^{1 / q n} \leq 1
$$

and it follows from (2.5) in Lemma 2, the relation $\mathrm{d}_{p n}=e^{p n+o(n)}$ and to (3.5) that this last inequality is satisfied for all $m \gg_{a, b, \kappa, H} 1$ because it is implied by the stronger inequality $b^{\kappa} e^{\kappa} \rho<1$, which holds by hypothesis. Hence, (3.4) holds with our choice of $n$ for large $m$.

We define

$$
\widehat{L}(\kappa, a, b)=\frac{\kappa+\kappa \log (b)+\log (\beta)}{(\kappa-1) \log (b)} .
$$

Let us fix $\varepsilon>0$. We deduce from (3.2) and (3.4) that, for all $m \geq$ $M_{1}(\varepsilon, \kappa, H, a, b)$ (it would in fact be possible to give an explicit bound), we have

$$
\begin{gathered}
-\log _{b}\left|\log \left(1-\frac{a}{b}\right)-\frac{u}{b^{m}}\right| \leq m+\left(\delta+\frac{\kappa-1}{2} \varepsilon\right)\left(\left\lfloor\frac{m}{q(\kappa-1)}\right\rfloor+1\right) q \\
\leq\left(1+\frac{\delta}{\kappa-1}+\frac{\varepsilon}{2}\right) m+\left(\delta+\frac{\kappa-1}{2} \varepsilon\right) q=(\widehat{L}(\kappa, a, b)+\varepsilon) m
\end{gathered}
$$

where the last equality holds provided that

$$
m \geq \max \left(M_{1}(\varepsilon, \kappa, H, a, b), \frac{2 \delta+\kappa-1}{\varepsilon} q\right)=M_{2}(\varepsilon, \kappa, H, a, b) .
$$

Second case. We now turn our attention to the case $(p-q) n<m$, which may happen in particular when $p=q$. We have

$$
\begin{aligned}
\left|\log \left(1-\frac{a}{b}\right)-\frac{u}{b^{m}}\right| & \geq\left|\frac{u}{b^{m}}-\frac{U_{n}}{b^{(p-q) n} V_{n}}\right|-\left|\log \left(1-\frac{a}{b}\right)-\frac{U_{n}}{b^{(p-q) n} V_{n}}\right| \\
& \geq \frac{1}{b^{m}\left|V_{n}\right|}-\left|\frac{R_{p n, q n}(a / b)}{B_{p n, q n}(a / b)}\right| .
\end{aligned}
$$


Hence, provided that

$$
\left|2 b^{m} V_{n} R_{p n, q n}(a / b)\right| \leq\left|B_{p n, q n}(a / b)\right|
$$

we have

$$
\left|\log \left(1-\frac{a}{b}\right)-\frac{u}{b^{m}}\right| \geq \frac{1}{2 b^{m}\left|V_{n}\right|} .
$$

As in the first case, since $V_{n} \rightarrow+\infty$, we need to choose $n$ as small as possible satisfying $(p-q) n<m$ and (3.7). We rewrite (3.7) as

$$
2 b^{m / q n} b \mathrm{~d}_{p n}^{1 / q n}\left|R_{p n, q n}(a / b)\right|^{1 / q n} \leq 1 .
$$

To find an optimal value of $n$ satisfying (3.9), we note that the condition $b^{\kappa} e^{\kappa} \rho<1$ implies that

$$
-\log \left(b e^{\kappa} \rho\right)>(\kappa-1) \log (b) \geq 0
$$

and therefore, for all $m \geq 1$,

$$
\frac{m}{q(\kappa-1)}>N=\frac{m \log (b)}{-q \log \left(b e^{\kappa} \rho\right)} \geq 0
$$

We note that we have $b^{m / q N} b e^{\kappa} \rho=1$. Hence, the integer $n$ defined by

$$
n=\lfloor N\rfloor+1=\left\lfloor\frac{m \log (b)}{-q \log \left(b e^{\kappa} \rho\right)}\right\rfloor+1
$$

satisfies $b^{m / q n} b e^{\kappa} \rho<1$ and also $(p-q) n<m$ if $m \gg_{\kappa, H, a, b} 1$ (from (3.10)).

Thus (3.9) is also satisfied for large enough $m \gg_{\kappa, H, a, b} 1$ and with the definition (3.11) of $n$, the lower bound (3.8) holds.

Let us fix $\varepsilon>0$. We deduce from (3.2) and (3.8) that, for all $m \geq$ $M_{3}(\varepsilon, \kappa, H, a, b)$,

$$
-\log _{b}\left|\log \left(1-\frac{a}{b}\right)-\frac{u}{b^{m}}\right| \leq m+\left(\delta-\frac{\log \left(b e^{\kappa} \rho\right)}{\log (b)} \frac{\varepsilon}{2}\right)\left(\left\lfloor\frac{m \log (b)}{-q \log \left(b e^{\kappa} \rho\right)}\right\rfloor+1\right) q .
$$

$$
\leq\left(1-\frac{\delta \log (b)}{\log \left(b e^{\kappa} \rho\right)}+\frac{\varepsilon}{2}\right) m+\left(\delta-\frac{\log \left(b e^{\kappa} \rho\right)}{\log (b)} \frac{\varepsilon}{2}\right) q=(L(\kappa, a, b)+\varepsilon) m
$$

where the last equality holds provided that

$$
m \geq \max \left(M_{3}(\varepsilon, \kappa, H, a, b), \frac{2 \delta-\frac{\log \left(b e^{\kappa} \rho\right)}{\log (b)} \varepsilon}{\varepsilon} q\right)=M_{4}(\varepsilon, \kappa, H, a, b) .
$$


Comparison of the two cases. We now prove that we have $\widehat{L}(\kappa, a, b) \geq$ $L(\kappa, a, b)$ for any given $\kappa \in \mathscr{L}(a, b), \kappa>1$. For simplicity, we define $X=\beta / \rho$, $Y=b^{\kappa-1}$ and $Z=\left(b^{\kappa} e^{\kappa} \rho\right)^{-1}$. We note that, for any $\kappa \in \mathscr{L}(a, b), \kappa>1$, we have $X>1, Y>1, Z>1$ and

$$
\begin{aligned}
\widehat{L}(\kappa, a, b)-L(\kappa, a, b) & =\frac{\log \left(b^{\kappa} e^{\kappa} \beta\right)}{\log \left(b^{\kappa-1}\right)}-\frac{\log (\beta / \rho)}{\log \left(\left(b e^{\kappa} \rho\right)^{-1}\right)} \\
= & \frac{\log (X / Z)}{\log (Y)}-\frac{\log (X)}{\log (Y Z)}=\frac{\log (Y Z) \log (X / Z)-\log (X) \log (Y)}{\log (Y) \log (Y Z)} .
\end{aligned}
$$

The denominator of the right hand side is clearly positive and it remains to prove that this is also the case of the numerator. Let $x, y, z$ be any real number $\geq 1$ : by calculus, we prove that $\log (y z) \log (x / z)-\log (x) \log (y) \geq 0$ if $z \in[1, x / y]$. To prove that $\widehat{L}(\kappa, a, b) \geq L(\kappa, a, b)$, it is therefore enough to check that $1 \leq Z \leq X / Y$ and, indeed, this is true because 1$)$ we already know that $Z>1$ and 2$)$ we have $X /(Y Z)=b e^{\kappa} \beta>1$ since $b \beta \geq 1$.

Thus, for all $m \geq M_{5}=\max \left(M_{2}, M_{4}\right)(\varepsilon, \kappa, H, a, b)$, we have

$$
-\log _{b}\left|\log \left(1-\frac{a}{b}\right)-\frac{u}{b^{m}}\right| \leq(L(\kappa, a, b)+\varepsilon) m .
$$

Since we can find $\kappa_{0} \in \mathscr{L}(a, b)$ (depending on $\left.\varepsilon, a, b\right)$ such that $L\left(\kappa_{0}, a, b\right) \leq$ $\Lambda(a, b, 1)+\varepsilon$, we finally obtain

$$
-\log _{b}\left|\log \left(1-\frac{a}{b}\right)-\frac{u}{b^{m}}\right| \leq(\Lambda(a, b, 1)+2 \varepsilon) m
$$

for all $\left.m \geq M_{5}\left(\varepsilon, \kappa_{0}, H\left(\kappa_{0}\right), a, b\right)\right)=M_{6}(\varepsilon, a, b)$.

Since $\Lambda(a, b, c)=\Lambda(a c, b c, 1)$, the proof of Theorem 1 is complete.

\section{Comparison with previous bounds}

It is interesting to compare the bound $L(\kappa, a c, b c)$ (which really depends on the special shape of $b^{m}$ ) with the general irrationality measure obtained using the same approximations: the linear form

$$
\begin{aligned}
\mathrm{d}_{p n} b^{p n} R_{p n, q n}(a / b)= & \mathrm{d}_{p n} b^{p n} B_{p n, q n}(a / b) \log \left(1-\frac{a}{b}\right) \\
& -\mathrm{d}_{p n} b^{p n} A_{p n, q n}(a / b) \in \mathbb{Z} \log \left(1-\frac{a}{b}\right)+\mathbb{Z}
\end{aligned}
$$

tends to 0 as $n \rightarrow+\infty$ exactly under the condition $b^{\kappa} e^{\kappa} \rho<1$, i.e. $\kappa \in$ $\mathscr{L}(a, b)$. We then use a classical criterion (see [2, Lemma 3] for example) to obtain

$$
-\log _{v}\left|\log \left(1-\frac{a}{b}\right)-\frac{u}{v}\right| \leq-\frac{\log (\beta / \rho)}{\log \left(b^{\kappa} e^{\kappa} \rho\right)}+\varepsilon=\mu(\kappa, a, b)+\varepsilon
$$

for all $\varepsilon>0$, all $u \in \mathbb{Z}$ and $v \gg_{\varepsilon, \kappa, a, b} 1$. 
Let $\mu(a, b)$ denote the infimum of $\mu(\kappa, a, b)$ over $\mathscr{L}(a, b)$, which is greater than 2 because of the convergents of $\log (1-a / b)$. It seems that $\mu(a, b)$ is always attained at $\kappa=1$ but we did not try to prove this.

Obviously, for all $\kappa \in \mathscr{L}(a, b)$, we have $L(\kappa, a, b) \leq \mu(\kappa, a, b)$ and therefore, for $c=1$, we have $\Lambda(a, b, 1) \leq \mu(a, b)$. For $c \geq 2$, we cannot rule out the possibility that $\Lambda(a, b, c)>\mu(a, b)$ since this inequality holds if $c \gg_{a, b} 1$ and hence in applications, it will be necessary to check which is the smallest of the two.

\section{Proof of Theorem 2}

We first need a simple upper bound for $\rho(\kappa, x)$ when $0<|x|<1$ and for this, we consider two separate cases.

Assume that $-1<x<0$. Then, for all $t \in(0,1), \frac{t^{\kappa}(1-t)}{1-x t} \leq t^{\kappa}(1-t)$, which implies that

$$
\rho(\kappa, x) \leq \frac{\kappa^{\kappa}}{(\kappa+1)^{\kappa+1}}|x|^{\kappa+1} .
$$

Assume that $0<x<1$. For all $t \in(0,1)$, we have $(1-x t)^{2} \geq(1-x)(1-t)$ and hence

$$
\frac{t^{\kappa}(1-t)}{1-x t} \leq \frac{t^{\kappa}(1-t)^{1 / 2}}{(1-x)^{1 / 2}}
$$

which implies that

$$
\rho(\kappa, x) \leq \frac{(2 \kappa)^{\kappa}}{(2 \kappa+1)^{\kappa+1 / 2}} \frac{|x|^{\kappa+1}}{(1-x)^{1 / 2}}
$$

We shall use the simpler strict bounds : $\rho(\kappa, x)<|x|^{\kappa+1}$ if $-1<x<0$ and $\rho(\kappa, x)<|x|^{\kappa+1}(1-x)^{-1 / 2}$ if $0<x<1$.

We also need an upper bound for $\beta(\kappa, x)$ : by definition, we have

$$
\beta(\kappa, x)=\frac{1}{|x|}\left(\min _{r>0} \frac{(1+|x| r)^{p}(1-x+|x| r)^{q}}{r^{q}}\right)^{1 / q} \leq 2^{\kappa}(2-x) .
$$

We get the last inequality by using the special value $r=1 /|x|$. We are now ready to prove the theorem. Let us suppose first that $-1<a / b<0$ and set $K=\log (b /|a|) /(1+\log |a|)$ : the condition $1+2 \log |a| \leq \log (b)$ ensures that $K \geq 1$ and we have

$$
b^{K} e^{K} \rho(K, a / b)<b^{K} e^{K}|a / b|^{K+1}=1 .
$$

Thus $K \in \mathscr{L}(a, b)$. 
Let us now suppose that $0<a / b<1$ and let

$$
k=\frac{\log (b / a)+\log \sqrt{1-a / b}}{1+\log (a)} .
$$

The condition $1+2 \log (a) \leq \log (b)+\log \sqrt{1-a / b}$ ensures that $k \geq 1$ and we have $b^{k} e^{k} \rho(k, a / b)<b^{k} e^{k}(a / b)^{k+1}(1-a / b)^{-1 / 2}=1$. Thus, once more, $k \in \mathscr{L}(a, b)$. Clearly,

$$
\Lambda(a, b, 1)=\inf _{\kappa \in \mathscr{L}(a, b)} L(\kappa, a, b) \leq\left\{\begin{array}{lcc}
L(K, a, b) & \text { if } \quad a<0 \\
L(k, a, b) & \text { if } & a>0 .
\end{array}\right.
$$

When $a<0$, we have

$$
\begin{aligned}
& L(K, a, b)=1+\frac{\log \beta(K, a / b)+K+\log (b)}{-(}\log \rho(K, a / b)+K+\log (b)) \\
& \quad \leq 1-\frac{\log \left(2^{K}(2-a / b)\right)+K+\log (b)}{\log \left(K^{K}(K+1)^{-K-1}|a / b|^{K+1}\right)+K+\log (b)}
\end{aligned}
$$

as desired, provided that the denominator of the right hand side is positive, i.e. that

$$
\frac{K^{K}}{(K+1)^{K+1}}\left|\frac{a}{b}\right|^{K+1} e^{K} b<1 .
$$

But, independently of the definition of $K$, this inequality is just a consequence of the hypothesis that $1+2 \log |a|<\log (b)$ :

$$
\left|\frac{a}{b}\right|^{K+1} e^{K} b=|a|\left|\frac{a e}{b}\right|^{K}<\frac{1}{|a|^{K-1}}<1 .
$$

(The factor $K^{K}(K+1)^{-K-1}<1$ has been removed for clarity.)

When $a>0$, we have

$$
\begin{aligned}
& L(k, a, b)=1+\frac{\log \beta(k, a / b)+k+\log (b)}{-(\log \rho(k, a / b)+k+\log (b))} \\
& \quad \leq 1-\frac{\log \left(2^{k}(2-a / b)\right)+k+\log (b)}{\log \left((2 k)^{k}(2 k+1)^{-k-1 / 2}(a / b)^{k+1}(1-a / b)^{-1 / 2}\right)+k+\log (b)}
\end{aligned}
$$

again provided that the denominator of the right hand side is positive, i.e. that

$$
\frac{(2 k)^{k}}{(2 k+1)^{k+1 / 2}}\left(\frac{a}{b}\right)^{k+1}(1-a / b)^{-1 / 2} e^{k} b<1
$$


This inequality is now a consequence of the hypothesis that $1+2 \log (a)<$ $\log (b)+\log \sqrt{1-a / b}$ :

$$
\begin{aligned}
(a / b)^{k+1} e^{k} b(1-a / b)^{-1 / 2} & =a(1-a / b)^{k-1 / 2}\left(\frac{a e}{b(1-a / b)^{1 / 2}}\right)^{k} \\
& <\frac{(1-a / b)^{k-1 / 2}}{a^{k-1}}<1 .
\end{aligned}
$$

(The factor $(2 k)^{k}(2 k+1)^{-k-1 / 2}<1$ has been removed for clarity.) The asymptotic expansions of these upper bounds for $\Lambda(a, b, 1)$ is a tedious exercise which can be done using a computer algebra. Finally, the theorem follows on replacing $a$ by $a c$ and $b$ by $b c$.

\section{Proof of Theorem 4}

In this section, we provide a sketch of the proof of Theorem 4 because it is very similar to that of Theorem 1.

(i) We consider the approximations provided by Lemma 1 with $p=q=1$ and $\kappa=1$. We define $v_{n}=b^{n} B_{n, n}(a / b) \in \mathbb{Z}$ and $u_{n}=\mathrm{d}_{n} b^{n} A_{n, n}(a / b) \in \mathbb{Z}$. By the procedure described in the proof of Theorem 1, we can assume that $u / \mathrm{d}_{m} \neq u_{n} /\left(\mathrm{d}_{n} v_{n}\right)$ and we have

$$
\begin{aligned}
\left|\log \left(1-\frac{a}{b}\right)-\frac{u}{\mathrm{~d}_{m}}\right| \geq \mid \frac{u}{\mathrm{~d}_{m}}- & \frac{u_{n}}{\mathrm{~d}_{n} y_{n}}|-| \log \left(1-\frac{a}{b}\right)-\frac{u_{n}}{\mathrm{~d}_{n} v_{n}} \mid \\
& \geq \frac{1}{\mathrm{~d}_{m}\left|v_{n}\right|}-\left|\frac{R_{n, n}(a / b)}{B_{n, n}(a / b)}\right| \quad \text { for all } m \geq n .
\end{aligned}
$$

Hence, if

$$
2 \mathrm{~d}_{m} b^{n} R_{n, n}(a / b) \leq 1
$$

we have

$$
\left|\log \left(1-\frac{a}{b}\right)-\frac{u}{\mathrm{~d}_{m}}\right| \geq \frac{1}{2 \mathrm{~d}_{m}\left|v_{n}\right|} .
$$

We now remark that the proof of Lemma 2 yields the following results: there exist two constants $c_{1}=c_{1}(a, b)$ and $c_{2}=c_{2}(a, b)$ such that, for all $n \geq 0$,

$$
\left|R_{n}(a / b)\right| \leq c_{1} \rho(1, a / b)^{n} \quad \text { and } \quad\left|B_{n}(a / b)\right| \leq c_{2} \beta(1, a / b)^{n}
$$

Condition (6.1) is satisfied if the stronger inequality $2 c_{1} \mathrm{~d}_{m} b^{n} \rho^{n} \leq 1$ holds: the smallest integer $N$ which satisfies this is

$$
N=\left\lfloor\frac{\log \left(2 c_{1} \mathrm{~d}_{m}\right)}{-\log (b \rho)}\right\rfloor+1
$$

Since we have supposed that $e b \rho<1$, we also have $-\log (b \rho)>1$ and therefore $0<N \leq m$ for large $m$. 
Hence (6.2) holds with $n=N$ and we have

$$
\left|\log \left(1-\frac{a}{b}\right)-\frac{u}{\mathrm{~d}_{m}}\right| \geq \frac{1}{2 \mathrm{~d}_{m}\left|v_{N}\right|} \geq \frac{1}{2 c_{2} \mathrm{~d}_{m} \beta^{N}} \geq \frac{\left(2 c_{1} \beta\right)^{1 / \log (b \rho)}}{2 c_{2} \mathrm{~d}_{m}^{-\log (\beta / \rho) / \log (b \rho)}} .
$$

It remains to note that $\beta(1, a / b) \rho(1, a / b)=|a / b|^{2}$.

(ii) To simplify, let $\ell=\lfloor\nu m\rfloor, x_{n}=\mathrm{d}_{n} b^{n} A_{p n, q n}(a / b) \in \mathbb{Z}$ and $y_{n}=$ $b^{n} B_{p n, q n}(a / b) \in \mathbb{Z}$. Like in the proof of Theorem 1 , we can assume that $u /\left(\mathrm{d}_{\ell} b^{m}\right) \neq x_{n} /\left(\mathrm{d}_{n} b^{(p-q) n} y_{n}\right)$ and we have

$$
\begin{aligned}
\mid \log \left(1-\frac{a}{b}\right) & -\frac{u}{d_{\ell} b^{m}}|\geq| \frac{u}{\mathrm{~d}_{\ell} b^{m}}-\frac{x_{n}}{\mathrm{~d}_{n} b_{n}^{(p-q) n} y_{n}} \mid \\
& -\left|\log \left(1-\frac{a}{b}\right)-\frac{x_{n}}{\mathrm{~d}_{n} b^{(p-q) n} y_{n}}\right| \geq \frac{1}{\mathrm{~d}_{\ell} b^{m}\left|y_{n}\right|}-\left|\frac{R_{p n, q n}(a / b)}{B_{p n, q n}(a / b)}\right|
\end{aligned}
$$

provided that $\ell \geq p n$ and $m \geq(p-q) n$. Hence, under these two assumptions and with

$$
2 \mathrm{~d}_{\ell} b^{m} b^{q n} R_{p n, q n}(a / b) \leq 1
$$

we have

$$
\left|\log \left(1-\frac{a}{b}\right)-\frac{u}{\mathrm{~d}_{\ell} b^{m}}\right| \geq \frac{1}{2 \mathrm{~d}_{\ell} b^{m}\left|y_{n}\right|}
$$

The integer

$$
n=\left\lfloor\frac{(\nu+\log (b)) m}{-q \log (b \rho)}\right\rfloor+1
$$

satisfies (6.3) for large enough $m$. Since $p / q \in \mathscr{D}(a, b, \nu)$, we have 1$)$ necessarily $-\log (b \rho)>0$ and therefore $n>0$ and 2) the conditions $\ell \geq p n$ and $m \geq(p-q) n$ are also satisfied. We conclude exactly as in the second case of the proof of Theorem 1.

To prove that $D(a c, b c, \nu) \rightarrow 1$ as $b \rightarrow+\infty$, we use the same method as in Theorem 2. Finally, (iii) is a consequence of $(i i)$.

\section{Further results}

For values of $a / b$ close to 1 , the value of $\Lambda(a, b, 1)$ may be greater than certain refined irrationality measures for $\log (1-a / b)$ : this is the case for $\log (2)$ whose best irrationality measure, due to Rukhadze [19], is:

$$
\left|\log (2)-\frac{p}{q}\right| \geq \frac{1}{q^{3.891310}}, \quad p \in \mathbb{Z}, \quad q \gg 1 .
$$

A list of such improvements is available in [13, p. 186]. 
In order to extend Theorem 3, it would be interesting to see if, using Rukhadze's method (in the generalised form of $[12,13]$ or (which in this case amounts to the same thing here) using the "group structure" integral method of Rhin-Viola [17]), it is possible to greatly improve our bounds and obtain, for example, $\Lambda(1, b, 1)<2$ for smaller values of $b$.

There exist many examples of formal power series $F$ for which we can compute part of the Padé table $\left([p / q]_{F}\right)_{p, q \geq 0}$, or even sometimes the complete table. For example, let us consider the Lerch function

$$
\Phi(x, 1, \alpha)=\sum_{k=0}^{\infty} \frac{x^{k}}{k+\alpha},
$$

for any rational $\alpha \geq 0$. As in the case of $\log (1-x)=-x \Phi(x, 1,0)$, the upper part $([p / q])_{p \geq q \geq 0}$ of the Padé table of $\Phi(x, 1, \alpha)$ is known (the proof is a simple adaptation of Lemma 1, see also [11]) and one obtains results similar to our Theorems 1, 2 and 3. We will state these results only in the particular case $\alpha=1 / 2$ which is of interest because the function

$$
x \Phi\left(x^{2}, 1,1 / 2\right)=\log \left(\frac{1+x}{1-x}\right),
$$

evaluated at $x=a /(2 b+a)$, produces an irrationality measure which complements Theorem 1: for all $\varepsilon>0$, all $u \in \mathbb{Z}$ and all $m \gg_{\varepsilon, a, b, c} 1$,

$$
\left|\log \left(1-\frac{a}{b}\right)-\frac{u}{(2 b c+a c)^{2 m}}\right| \geq \frac{1}{(2 b c+a c)^{2 m(N(a c, b c)+\varepsilon)}},
$$

where

$$
N(a, b)=\inf _{\kappa \in \mathscr{T}(A, B)} \frac{\log (\beta(\kappa, A / B) / \rho(\kappa, A / B))}{-\log \left(4 B e^{2 \kappa} \rho(\kappa, A / B)\right)},
$$

with $A=a^{2}, B=(2 b+a)^{2}$ and $\mathscr{T}(A, B)=\left\{\kappa \geq 1: 4 B^{\kappa} e^{2 \kappa} \rho(\kappa, A / B)<1\right\}$. Clearly, $N(a, b)=N(a-2, b+1)$. We have for example

$$
\left|\log (12 / 13)-\frac{u}{729^{m}}\right| \geq \frac{1}{729^{1.969329 m}} .
$$

The function $x \Phi\left(-x^{2}, 1,1 / 2\right)=2 \arctan (x)$ is also an interesting special case because of its connection with the number $\pi$; however, the author found no measure of the shape $\left|\pi-u / b^{m}\right| \geq b^{-\mu(\pi) m}$ with $\mu(\pi)<2$ for at least one value of $b \geq 2$.

Alternatively, we could also obtain improved lower bounds like

$$
\left|\log \left(1-\frac{a}{b}\right)-\frac{u}{b^{q n} B_{p n, q n}(a / b)}\right| \geq \frac{1}{\left(b^{q n} B_{p n, q n}(a / b)\right)^{V(a, b)}}
$$

with $V(a, b) \geq 1$, and similar results for $b^{p m} B_{p m, q m}(a / b), \mathrm{d}_{p m} b^{q m} B_{p m, q m}(a / b)$, etc, where $B_{p n, q n}(X)$ is the Padé polynomial given in (2.2). 
Such sequences could be modified endlessly and are not usually expressible in a closed and attractive form. It would be also interesting to generalise the simultaneous type II Padé approximants for the familly $\log \left(1-a_{1} x\right)$, $\log \left(1-a_{2} x\right), \ldots, \log \left(1-a_{k} x\right)$ considered by Rhin and Toffin in [16], in order to obtain a result similar to Theorem 1 for certain rational linear forms in logarithms.

Finally, we conclude this article by mentioning an example of a completely different nature. The complete Padé table of the exponential function is known explicitly (it was Padé's case-study [15]). The diagonal Padé approximants $[n / n]_{\exp }$ contains a lot of diophantine information, for it is well-known that $[n / n]_{\exp }$ evaluated at a rational of the form $1 / b$ provide the convergents of $\exp (1 / b)$ and a very precise irrationality measure of $\exp (1 / b)$ : see $[1,9]$. Hence it would be natural to expect that $[p / q]_{\exp }$ could be used to obtain irrationality measures with denominators of the shape $b^{m}$. However, very surprisingly, this is not the case and we can not obtain something better: the growth of the denominator and remainder of $[p n / q n]_{\exp }$ behave like $C_{1}(p, q)^{n}(p n !)$ and $C_{2}(p, q)^{n} /((p+q) n)$ ! respectively, which completely annihilates the effect of the term $b^{(p-q) n}$, which was crucial in the proof of Theorem 1.

\section{References}

[1] Adams, W. W.: Asymptotic diophantine approximations to e. Proc. Nat. Acad. Sci. USA 55 (1966), 28-31.

[2] Alladi, K. And Robinson, M. L.: Legendre polynomials and irrationality. J. Reine Angew. Math. 318 (1980), 137-155.

[3] Bailey, D. H., Borwein, J. M., Crandall R. and Pomerance, C.: On the binary expansions of algebraic numbers. J. Théor. Nombres Bordeaux 16 (2004), no. 3, 487-518.

[4] Baker, G. A. and Graves-Morris, P.: Padé approximants. Second edition. Encyclopedia of Mathematics and its Applications 59. Cambridge University Press, Cambridge, 1996.

[5] Bauer, M. And Bennett, M. A.: Applications of the hypergeometric method to the generalized Ramanujan-Nagell equation. Ramanujan J. 6 (2002), 209-270.

[6] Beukers, F.: On the generalized Ramanujan-Nagell equation. I. Acta Arith. 38 (1980/81), no. 4, 389-410.

[7] Beukers, F.: On the generalized Ramanujan-Nagell equation. II. Acta Arith. 39 (1981), no. 2, 113-123.

[8] Brent, R., van der Poorten, A. and te Riele, H. J. J.: A comparative study of algorithms for computing continued fractions of algebraic 
numbers. In Algorithmic number theory (Talence, 1996), 35-47. Lecture Notes in Comput. Sci. 1122. Springer, Berlin, 1996.

[9] Bundschun, P.: Irrationalitätsmaße für $e^{a}, a \neq 0$ rational oder LiouvilleZahl. Math. Ann. 192 (1971), 229-242.

[10] Dubitskas, A. K.: Approximation of some logarithms of rational numbers by rational fractions of special form. (Russian). Vestnik Moskov. Univ. Ser. I Mat. Mekh. (1990), no. 2, 69-71.

[11] Duverney, D.: Trois techniques de calcul explicit d'approximants de Padé. Revue de Mathématiques Spéciales 8 (1996), 711-722.

[12] Hata, M.: Legendre type polynomials and irrationality measures. J. Reine Angew. Math. 407 (1990), 99-125.

[13] Heimonen, A., Matala-Aho, T. and VäÄnänen, K.: On irrationality measures of the values of Gauss hypergeometric function. Manuscripta Math. 81 (1993), no. 1-2, 183-202.

[14] Jackson, T. And Matthews, K.: On Shanks' algorithm for computing the continued fraction of $\log _{b} a$. J. Integer Seq. 5 (2002), Article 02.2.7.

[15] PadÉ, H.: CEuvres. Edited by Claude Brezinski. Bibliothèque Scientifique Albert Blanchard. Librairie Scientifique et Technique Albert Blanchard, Paris, 1984.

[16] Rhin, G. And Toffin, P.: Approximants de Padé simultanés de logarithmes. J. Number Theory 24 (1986), no. 3, 284-297.

[17] Rhin, G. And Viola, C.: On a permutation group related to $\zeta(2)$. Acta Arith. 77 (1996), no. 1, 23-56.

[18] Ridout, D.: Rational approximations to algebraic numbers. Mathematika 4 (1957), 125-131.

[19] Rukhadze, E. A.: A lower bound for the approximation of $\ln 2$ by rational numbers. Vestnik Moskov. Univ. Ser. I Mat. Mekh. 97 (1987), no. 6, 25-29.

[20] Waldschmidt, M.: Diophantine approximation on linear algebraic groups. Transcendence properties of the exponential function in several variables. Grundlehren der Mathematischen Wissenschaften 326. SpringerVerlag, Berlin, 2000.

Recibido: 31 de enero de 2006

Revisado: 12 de septiembre de 2006

Tanguy Rivoal

Institut Fourier

CNRS et Université Grenoble 1 100 rue des Maths, BP 74

38402 Saint-Martin d'Hères cedex, France rivoal@ujf-grenoble.fr 